\title{
Twin - A Design Pattern for Modeling Multiple Inheritance
}

\author{
Hanspeter Mössenböck \\ University of Linz, Institute of Practical Computer Science, A-4040 Linz \\ moessenboeck@ssw.uni-linz.ac.at
}

\begin{abstract}
We introduce an object-oriented design pattern called Twin that allows us to model multiple inheritance in programming languages that do not support this feature (e.g. Java, Modula-3, Oberon-2). The pattern avoids many of the problems of multiple inheritance while keeping most of its benefits. The structure of this paper corresponds to the form of the design pattern catalogue in [GHJV95].
\end{abstract}

\section{Motivation}

Design patterns are schematic standard solutions to recurring software design problems. They encapsulate a designer's experience and makes it reusable in similar contexts. Recently, a great number of design patterns has been discovered and published ([GHJV95], [Pree95], [BMRSS96]). Some of them are directly supported in a programming language (e.g. the Prototype pattern in Self or the Iterator pattern CLU), some are not. In this paper we describe a design pattern, which allows a programmer to simulate multiple inheritance in languages which do not support this feature directly.

Multiple inheritance allows one to inherit data and code from more than one base class. It is a controversial feature that is claimed to be indispensable by some programmers, but also blamed for problems by others, since it can lead to name clashes, complexity and inefficiency. In most cases, software architectures become cleaner and simpler when multiple inheritance is avoided, but there are also situations where this feature is really needed. If one is programming in a language that does not support multiple inheritance (e.g. in Java, Modula-3 oder Oberon-2), but if one really needs this feature, one has to find a work-around. The Twin pattern-introduced in this paper-provides a standard solution for such cases. It gives one most of the benefits of multiple inheritance while avoiding many of its problems.

The rest of this paper is structured according to the pattern catalogue in [GHJV95] so that the Twin pattern could in principle be incorporated into this catalogue.

\subsection{Example}

As a motivating example for a situation that requires multiple inheritance, consider a computer ball game consisting of active and passive game objects. The active objects 
are balls that move across the screen at a certain speed. The passive objects are paddles, walls and other obstacles that are either fixed at a certain screen position or can be moved under the control of the user.

The design of such a game is shown in Fig. 1. All game items (paddles, walls, balls, etc.) are derived from a common base class GameItem from which they inherit methods for drawing or collision checking. Methods such as draw() and intersects() are abstract and have to be refined in subclasses. check() is a template method, i.e. it consists of calls to other abstract methods that must be implemented by concrete game item classes later. It tests if an item intersects with some other and calls the other item's collideWith() method in that case. In addition to being game items, active objects (i.e. balls) are also derived from class Thread. All threads are controlled by a scheduler using preemptive multitasking.

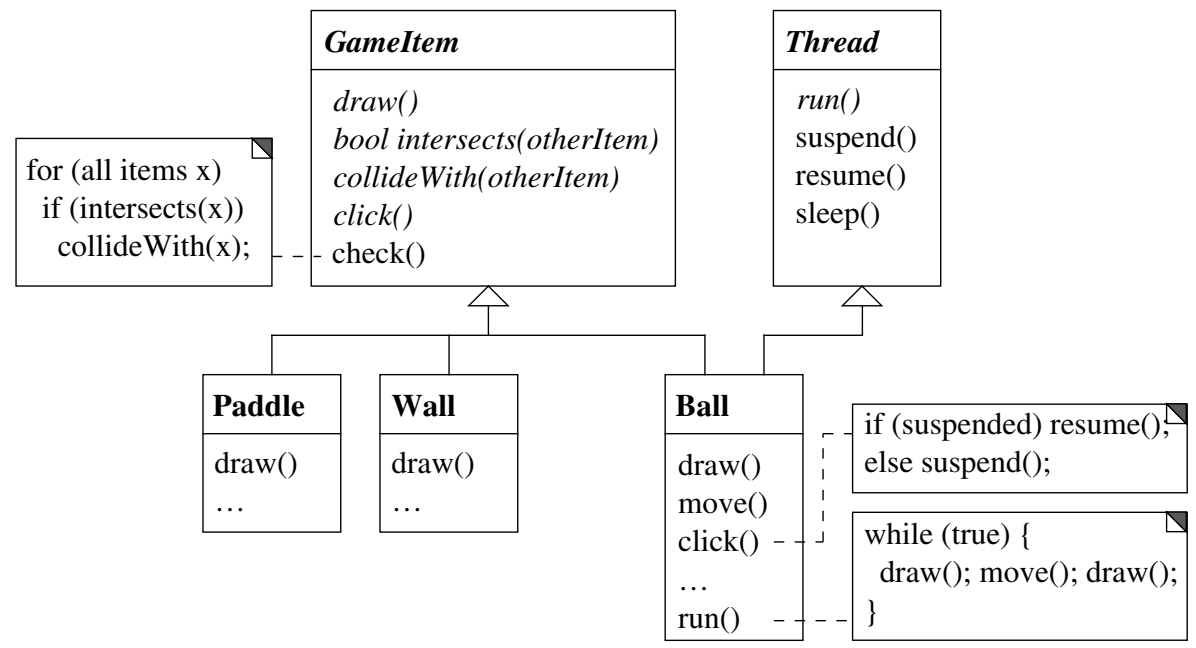

Fig. 1. Class hierarchy of a computer ball game

The body of a ball thread is implemented in its run() method. When a ball thread is running, it repeatedly moves and draws the ball. If the user clicks on a ball, the ball sends itself a suspend() message to stop its movement. Clicking on the ball again sends a resume() message to make the ball moving again.

The important thing about this example is that balls are both game items and threads (i.e. they are compatible with both). They can be linked into a list of game items, for example, so that they can be sent draw() and intersects() messages. But they can also be linked into a list of threads from which the scheduler selects the next thread to run. Thus, balls have to be compatible with both base classes. This is a typical case where multiple inheritance is useful.

Languages like Java don't support multiple inheritance, so how can we implement this design in Java? In Java, a class can extend only one base class but it can implement several interfaces. Let's see, if we can get along with multiple interface inheritance here. Ball could extend Thread and thus inherit the code of suspend() and resume(). However, it is not possible to treat GameItem just as an interface because GameItem is not fully abstract. It has a method check(), which contains code. Ball 
would like to inherit this code from GameItem and should therefore extend it as well. Thus Ball really has to extend two base classes.

This is the place where the Twin pattern comes in. The basic idea is as follows: Instead of having a single class Ball that is derived from both GameItem and Thread, we have two separate classes Ballitem and BallThread, which are derived from GameItem and Thread, respectively (Fig. 2). Ballitem and BallThread are closely coupled via fields so that we can view them as a Twin object having two ends: The Ballitem end is compatible with GameItem and can be linked into a list of game items; the BallThread end is compatible with Thread and can be linked into a list of threads.

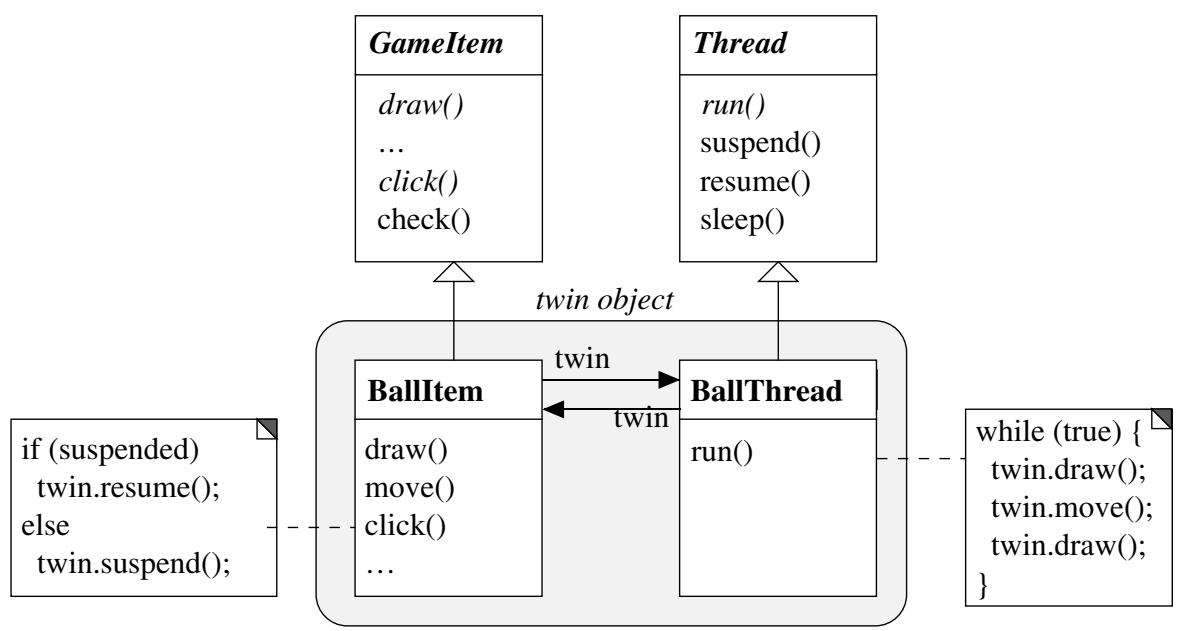

Fig. 2. The class Ball from Fig.1 was split into two classes, which make up a twin object

Twin objects are always created in pairs. When the scheduler activates a BallThread object by calling its method $\operatorname{run}()$, the object moves the ball by sending its twin the messages move() and $\operatorname{draw}()$. On the other hand, when the user clicks on a ball with the mouse, the Ballitem object reacts to the click and sends its twin the messages suspend() and resume() as appropriate.

Using only single inheritance, we have obtained most of the benefits of multiple inheritance: Active game objects inherit code from both GameItem and Thread. They are also compatible with both, i.e. they can be treated both as game items (draw, click) and as threads (run). As a pleasant side effect, we have avoided a major problem of multiple inheritance, namely name clashes. If GameItem and Thread had fields or methods with the same name, they would be inherited by Ballitem and BallThread independently. No name clash would occur. Similarly, if GameItem and Thread had a common base class $B$, the fields and methods of $B$ would be handed down to Ballttem and to BallThread separately—again without name clashes.

\section{Applicability}

The Twin pattern can be used 
- to simulate multiple inheritance in a language that does not support this feature.

- to avoid certain problems of multiple inheritance such as name clashes.

\section{Structure}

The typical structure of multiple inheritance is described in Fig.3.

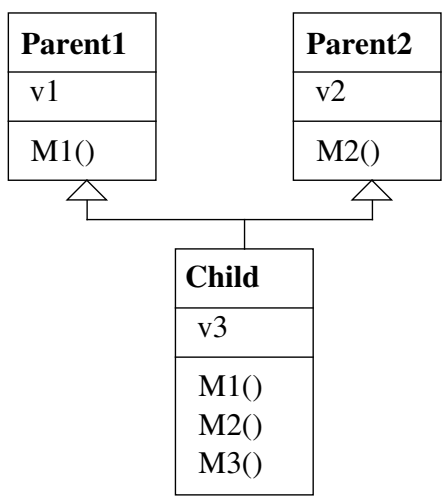

Fig. 3. Typical structure of multiple inheritance

It can be replaced by the Twin pattern structure described in Fig.4.

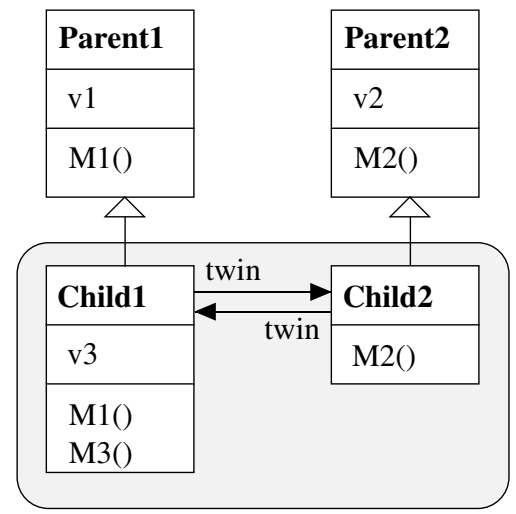

Fig. 4. Typical structure of the Twin pattern

\section{Participants}

Parent1 (GameItem) and Parent2 (Thread)

- The classes from which you want to inherit. 


\section{Child1 (BallItem) and Child2 (BallThread)}

- The subclasses of Parent1 and Parent2. They are mutually linked via fields. Each subclass may override methods inherited from its parent. New methods and fields are usually declared just in one of the subclasses (e.g. in Childl).

\section{Collaborations}

- Every child class is responsible for the protocol inherited from its parent. It handles messages from this protocol and forwards other messages to its partner class.

- Clients of the twin pattern reference one of the twin objects directly (e.g. ballitem) and the other via its twin field (e.g. ballitem.twin).

- Clients that rely on the protocols of Parent1 or Parent 2 communicate with objects of the respective child class (Child1 or Child2).

\section{Consequences}

Although the Twin pattern is able to simulate multiple inheritance, it is not identical to it. There are several problems that one has to be aware of:

1. Subclassing the Twin pattern. If the twin pattern should again be subclassed, it is often sufficient to subclass just one of the partners, for example Childl. In order to pass the interface of both partner classes down to the subclass, it is convenient to collect the methods of both partners in one class. One can add the methods of Child2 also to Child1 and let them forward requests to the other partner (Fig.5). 


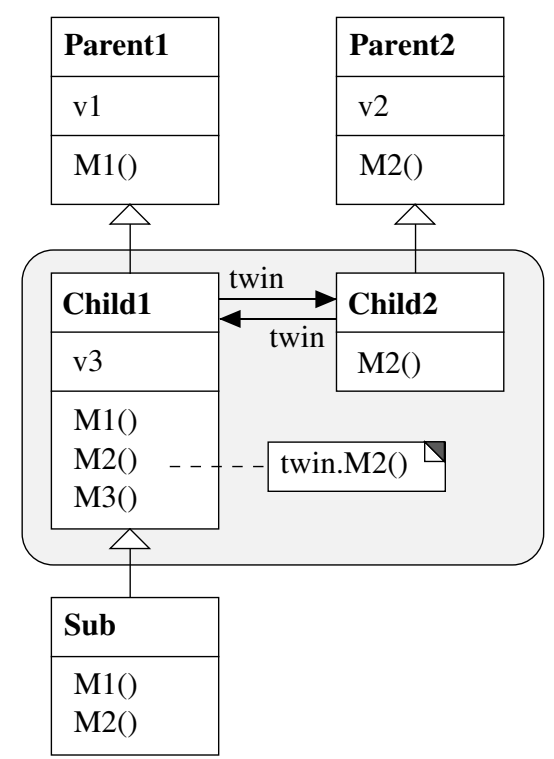

Fig. 5. Subclassing a twin class. Child1.M2() forwards the message to Child2.M2()

This solution has the problem that $S u b$ is only compatible with Childl but not with Child2. If one wants to make the subclass compatible with both Child1 and Child2 one has to model it according to the Twin pattern again (Fig.6).

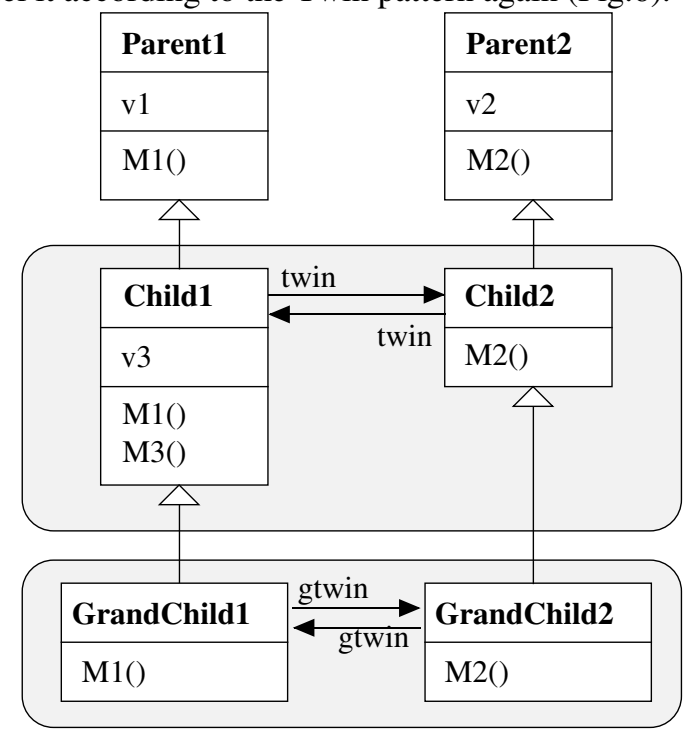

Fig. 6. The subclass of Childl and Child 2 is again a Twin class

2. More than two parent classes. The Twin pattern can be extended to more than two parent classes in a straightforward way. For every parent class there must be a child class. All child classes have to be mutually linked via fields (Fig.7). 


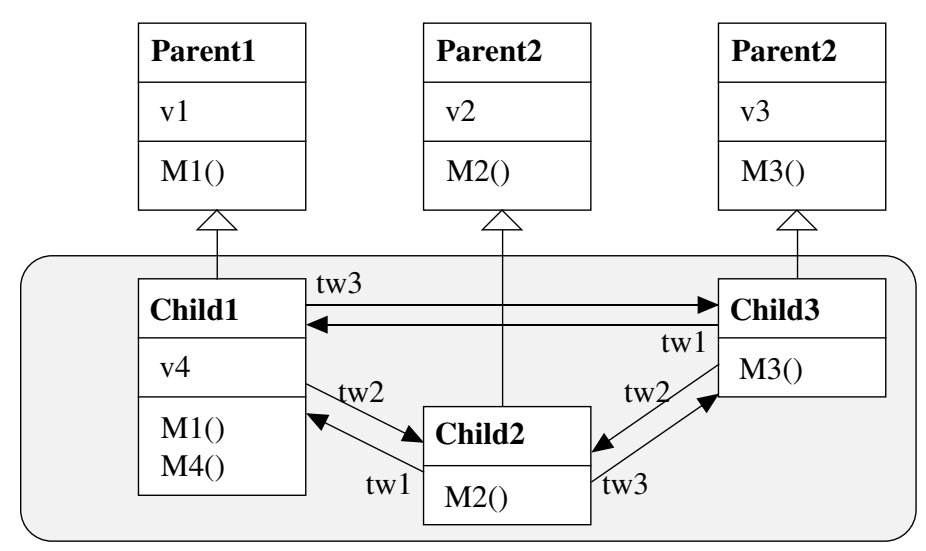

Fig. 7. A Twin class derived from three parent classes

Although this is considerably more complex than multiple inheritance, it is rare that a class inherits from more than two parent classes.

\section{Implementation}

The following issues should be considered when implementing the Twin pattern:

1. Data abstraction. The partners of a twin class have to cooperate closely. They probably have to access each others' private fields and methods. Most languages provide features to do that, i.e. to let related classes see more about each other than foreign classes. In Java, one can put the partner classes into a common package and implement the private fields and methods with the package visibility attribute. In Modula-3 and Oberon one can put the partner classes into the same module so that they have unrestricted access to each others' components.

2. Efficiency. The Twin pattern replaces inheritance relationships by composition. This requires forwarding of messages, which is less efficient than inheritance. However, multiple inheritance is anyway slightly less efficient than single inheritance [Str89] so that the additional run time costs of the Twin pattern are not a major problem.

\section{Sample Code}

We sketch the implementation of the motivating example (a computer game board with moving balls) in Java. The board is represented by a class GameBoard. It has a certain width and height and a reference to a list of game items. 


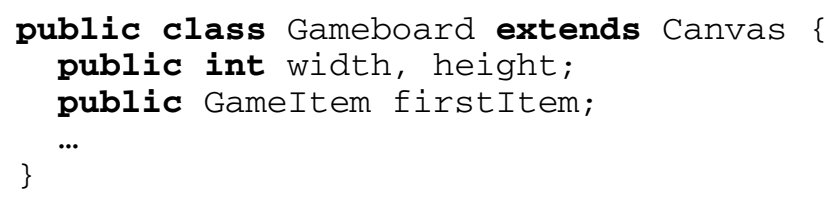

The game items are derived from an abstract class GameItem. Every item has a reference to the game board, a position on this board and a reference to the next game item. It has abstract methods to draw itself, to react on mouse clicks, to check whether it intersects with some other game item and to take measures for a collision with other game items. 


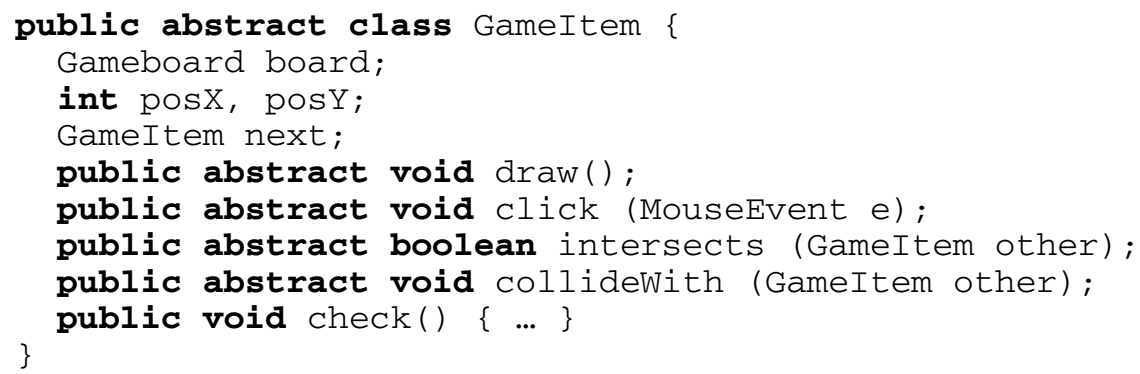

The method $\operatorname{check}()$ is a template method, which checks if this object intersects with any other object on the board. If so, it does whatever it has to do for a collision.

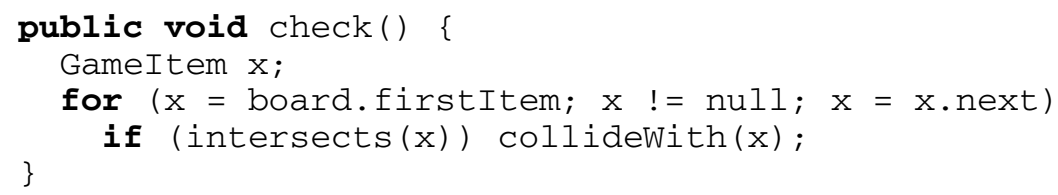

Balls are twin objects derived from GameItem and Thread. As shown in Fig. 2 we implement the twin group as Ballitem (a subclass of GameItem) and BallThread (a subclass of Thread). Ball items move at a certain speed $(d x, d y)$ and have to override the inherited methods draw, click, intersects and collideWith.

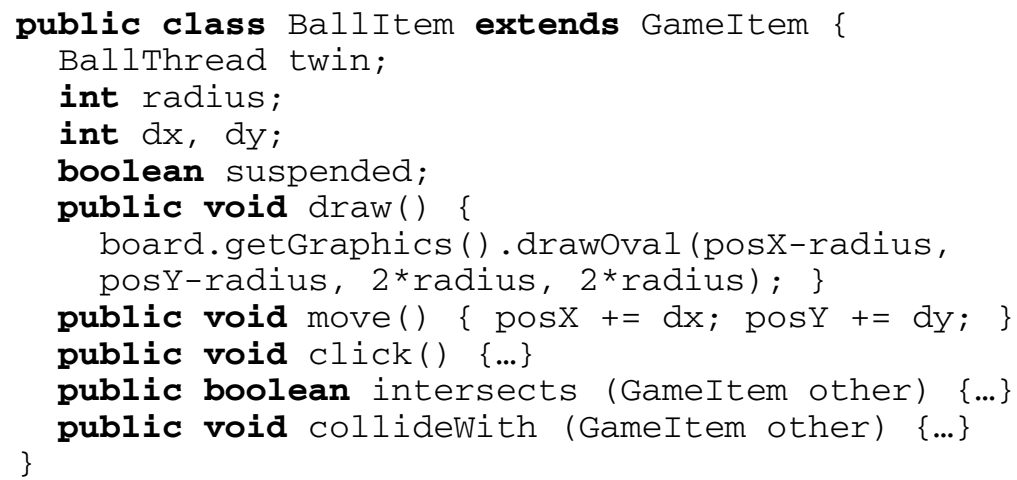

In order to simplify things, we assume that balls can only collide with walls, which are another kind of game items. The intersects method of a Ballitem can then be implemented as

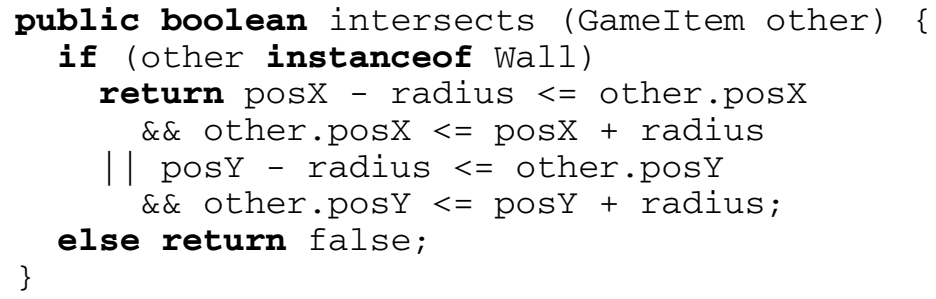

A collision with a wall changes the direction of the ball, which can be implemented as 


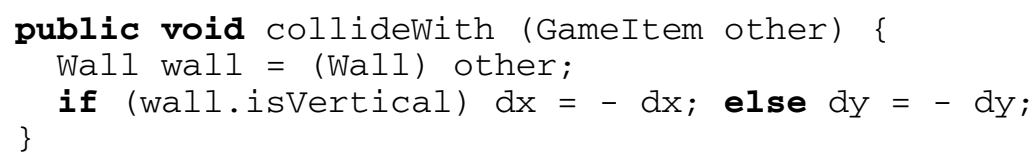

When the user clicks on a moving ball it stops; when he clicks on a stopped ball it starts to move again. This is implemented by suspending and resuming the corresponding ball thread (the twin object).

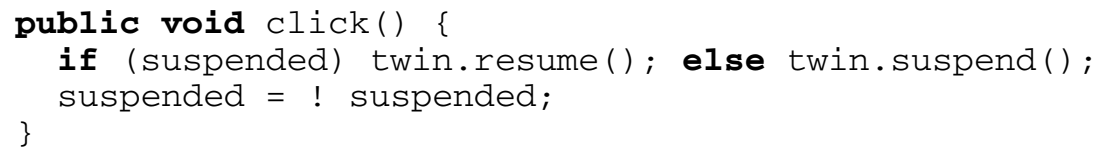

The class BallThread is derived from the standard class java.lang.Thread. It has a reference to its twin class Ballitem. The only method that has to be implemented is $\operatorname{run}()$. The implementation of other methods such as suspend() and resume() is inherited from Thread.

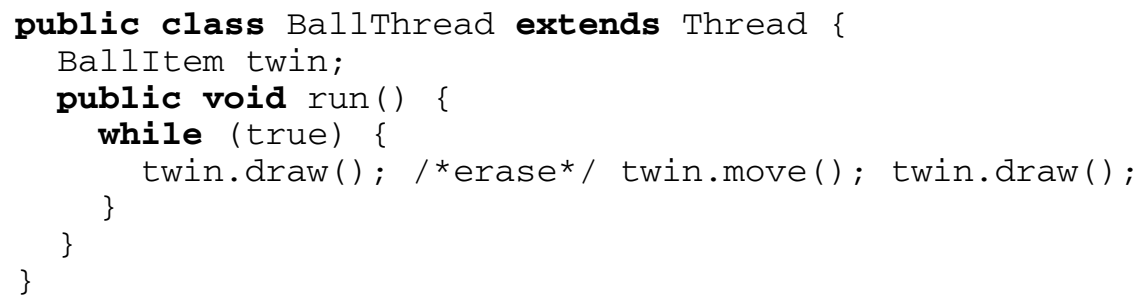

When a new ball is needed, the program has to create both a Ballitem and a BallThread object and link them together, for example: 


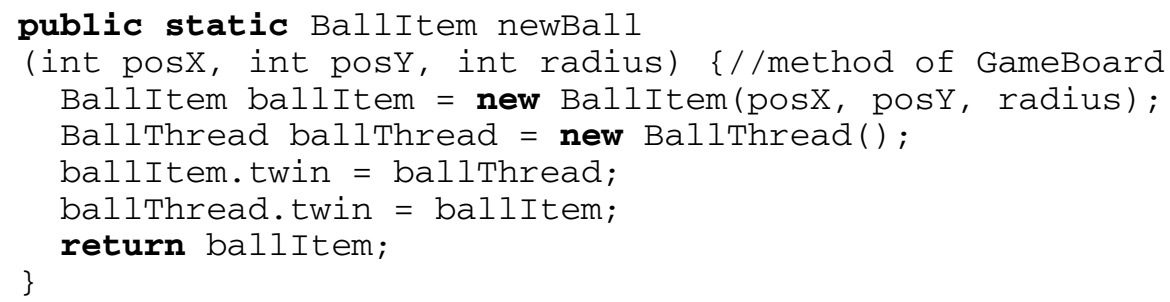

The returned ball item can be linked into the list of game items in the game board. The corresponding ball thread can be started to make the ball move.

\section{Known Uses}

The motivating example of a ball game (Section 1) was implemented as a teaching exercise in Oberon-2, a language that does not support multiple inheritance. The Oberon system uses cooperative multitasking. It maintains a list of user processes that are activated whenever the system is idle. A ball is a special instance of a process and at the same time a game object.

Another example can be found in the context of Java applets. Applets are active objects that live on Web pages and react on user input such as mouse clicks. When a user clicks on an applet, the applet notifies all registered mouse listeners to react on the event. If an applet wants to react on the click itself, it has to implement the MouseListener interface, so that it can be registered as an appropriate listener with itself. It must also extend the class Applet. The following code shows the declaration of a class MyApplet:

class MyApplet extends Applet implements MouseListener \{ \}

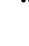

The MouseListener interface (a standard interface of the Java libraries) specifies 5 methods that have to be implemented in MyApplet:

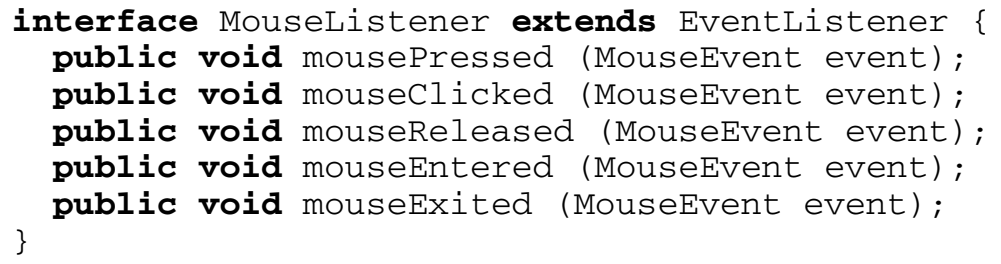

Some of these methods are often identical in different listener implementations. For example, several listeners change the shape of the cursor in the same way when it enters or exits the applet area on the screen. Therefore, we would like to have a prefabricated mouse listener class (StdMouseListener), which already provides standard implementations for the methods mouseEntered and mouseExited. Other listeners could then inherit these standard implementations. 
We are now in a situation where we would like to inherit code from two classes, namely from Applet and StdMouseListener, but this is not possible in Java. We can only inherit from one class. We can, however, apply the Twin pattern, which results in the following architecture (Fig.8).

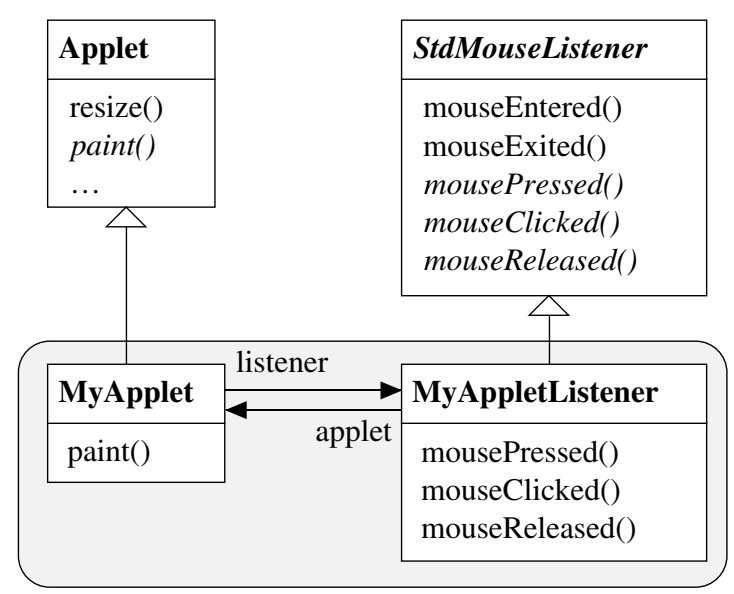

Fig. 8. A twin applet that inherits code both from Applet and from StdMouseListener

MyApplet inherits code from Applet; MyAppletListener inherits code from StdMouseListener. A MyAppletListener object will be registered as a mouse listener for MyApplet. When it is notified about a mouse click it accesses its applet to perform an appropriate action.

In [CaW98] a similar solution is presented using inner classes. MyAppletListener is implemented there as an inner class of MyApplet. This allows MyAppletListener to access all private instance variables of MyApplet. No explicit link between the classes is necessary. However, this solution is asymmetric. MyApplet cannot access the private instance variables of MyAppletListener.

\section{Related Patterns}

The Twin pattern is related to the Adapter pattern, especially to the Two-Way-Adapter described in [GHJV95], which is recommended when two different clients need to view an object differently. However, the Two-Way-Adapter is implemented with multiple inheritance while the Twin avoids this feature.

\section{Acknowledgements}

The technique described in this paper was discovered by Robert Griesemer in the implementation of a game program in Oberon. It was also described-although not as a design pattern-in [Tem93] and [Moe93]. 


\section{References}

[BMRSS96] Buschmann F., Meunier R., Rohnert H., Sommerlad P., Stal M.: Pattern-oriented Software Architecture: A System of Patterns. Wiley 1996.

[CaW98] Campione M., Walrath K.: The Java Tutorial, 2nd edition, Addison-Wesley, 1998.

[GHJV95] Gamma E., Helm R., Johnson R., Vlissides J.: Design Patterns - Elements of Reusable Object-Oriented Software. Addison-Wesley 1995.

[Moe93] Mössenböck H.: Objektorientierte Programmierung in Oberon-2. Springer-Verlag 1993.

[Pree95] Pree W.: Design Patterns for Object-Oriented Software Development. AddisonWesley 1995.

[Str89] Stroustrup B.: Multiple Inheritance for C++. Proceedings EUUG Spring Conference, Helsinki, May 1989.

[Tem93] Templ J.: A Systematic Approach to Multiple Inheritance Implementation. SIGPLAN Notices 28 (4): 61-66 